\title{
Retraction of: A Biomimetic Emu Oil-Blended Electrospun Nanofibrous Mat for Maintaining Stemness of Adipose Tissue-Derived Stem Cells, by Jalilzadeh-Tabrizi S, Pilehvar-Soltanahmadi Y, Alizadeh E, Alipour S, Dadashpour M, Nejati-Koshki K, and Zarghami N. Biopreservation and Biobanking 2018;16(2);66-76. DOI: 10.1089/bio.2017.0056
}

$\boldsymbol{B}$ iopreservation and Biobanking is officially retracting the article entitled, A Biomimetic Emu Oil-Blended Electrospun Nanofibrous Mat for Maintaining Stemness of Adipose Tissue-Derived Stem Cells, by Jalilzadeh-Tabrizi S, PilehvarSoltanahmadi Y, Alizadeh E, Alipour S, Dadashpour M, Nejati-Koshki K, and Zarghami N (Biopreservation and Biobanking 2018;16(2);66-76. DOI: 10.1089/bio.2017.0056) due to the submission of the same images to different journals for different purposes, but without any attribution, reference, or reproduction permission.

The article had at first been corrected to include an added reference, but that initiated a further assessment by the editorial leadership. The findings of that added evaluation proved that no permission had been sought or granted to reproduce previously published work, nor was there any stated acknowledgment that the image appeared in previously published material. Therefore, the decision was made to retract the article from the scientific literature.

Biopreservation and Biobanking is dedicated to upholding the highest standards of peer review and does not tolerate any improprieties. 